\section{International Scientific Journal Theoretical \& Applied Science}

\author{
p-ISSN: 2308-4944 (print) e-ISSN: 2409-0085 (online) \\ Year: $2016 \quad$ Issue: 4 Volume: 36 \\ Published: $30.04 .2016 \quad \underline{\text { http://T-Science.org }}$
}

SECTION 7. Mechanics and machine construction.
Seysen Zaurbekovich Kazakbaev

Candidate of technical Sciences, corresponding member RAM, Taraz innovation and humanities university, Kazakhstan $\underline{\text { seisen58@mail.ru }}$

Nurlan Syrymbayevich Karymsakov candidate of technical Sciences, senior lecturer, Taraz state University named after M.Kh.Dulati, Kazakhstan nurkar@mail.ru

Murat Musabekovich Bekmuratov Candidate of technical Sciences, Associate Professor, professor TARSU, Taraz state University named after M.Kh.Dulati, Kazakhstan

Alexandr Shevtsov Candidate of technical Sciences, member of PILA (USA), member of European Academy of Natural History (UK), Associate Professor, Taraz state University named after M.Kh.Dulati, Kazakhstan $\underline{\text { Shev_AlexXXXX@mail.ru }}$

Inessa Aleksandrovna Son 4th year student of the specialty mechanical engineering, Taraz state University named after M.Kh.Dulati,

Kazakhstan inna06.02@mail.ru

Violetta Aleksandrovna Son

3rd year student of the specialty processing machinery and eguipment, Taraz state University named after M.Kh.Dulati, Kazakhstan

\title{
THE GRAIN THROWER-CLASSIFIER FOR PRE-CLEANING GRAIN
}

Abstract: In the article the problems of creating high-performance and efficient machines on the basis of grain casters. Optimal parameters of technological operations for cleaning grain from various impurities. Development of the optimal design of pneumatic rotary classifier, which allows the combination of the transfer and primary processing preliminary drying of grain products.

Key words: cleaning impurities, post-harvest processing of grain, grain thrower-classifier PCL, innovative technique and technology of grain handling (ITPOS).

Language: Russian

Citation: Kazakbaev SZ, Karymsakov NS, Bekmuratov MM, Shevtsov AN, Son IA, Son VA (2016) THE GRAIN THROWER-CLASSIFIER FOR PRE-CLEANING GRAIN. ISJ Theoretical \& Applied Science, 04 (36): 76-82.

Soi: http://s-0-i.org/1.1/TAS-04-36-11 Doi: crossef http://dx.doi.org/10.15863/TAS.2016.04.36.11

\section{ЗЕРНОМЕТАТЕЛЬ-КЛАССИФИКАТОР ДЛЯ ПРЕДВАРИТЕЛЬНОЙ ОЧИСТКИ ЗЕРНА}

Аннотация: В работе рассматриваются проблемы по созданию высокопроизводительных и эффективных машин на базе Зернометателей ЗМ-60. Исследуются оптимальные параметры как технологической операции по очистке зерна от различных примесей, так и по разработке оптимальной конструкции пневмороторного классификатора, позволяющие совмещчение перегрузки и первичной переработки с предварительной сушкой зернопродуктов.

Ключевые слова: очистка примесей, послеуборочная обработка зерна, зернометательклассификатор ЗКС, инновационная техника и технологии послеуборочной обработке зерна (ИТТПОЗ). 
Производство зерна в сельском хозяйстве завершается послеуборочной обработкой. В общем производственном процессе возделывания, уборки и послеуборочной обработки урожая зерновых и других культур, основные затраты приходятся на послеуборочную обработку, заключающуюся в очистке, сушке и доведении до требуемых кондиций по чистоте, влажности и другим показателям зерна и семян.

Послеуборочная обработка - это ключевое звено в производстве зерна. От него зависит, насколько окупятся затраты на все предыдущие стадии цикла. Практический опыт показывает, что сохранение запасов зерна является процессом достаточно сложным и в значительной мере зависит от качества его послеуборочной обработки. В период уборки урожая на токах скапливается большое количество зерна с высокой влажностью и засорённостью. При хранении такого зерна начинают развиваться нежелательные биологические процессы, в результате которых происходит его самосогревание. Это приводит к полной негодности зерна.

Послеуборочная обработка имеет большое значение для сохранности зерна и семян длительное время. Она включает комплекс последовательных технологических операций, в результате которых улучшаются многие качественные показатели семян и зерна. Выделение примесей изменяет компонентный состав зерновой массы, её физические свойства. Таким образом, в конечном счете послеуборочная обработка зерна позволяет снизить потери и увеличить экономический эффект от производства продукции.

Своевременная и качественная уборка, а также послеуборочная обработка урожая зерновых культур затруднена из-за недостатка техники, дефицита рабочей силы и особенностей природно-климатических условий. Слабая оснащенность хозяйств зерноочистительной техникой и оборудованием для временной консервации свежеубранной зерновой массы в неблагоприятные годы приводит к тому, что материал в ожидании очистки и сушки длительное время может находиться в буртах на открытых площадках. Вследствие этого происходит самосогревание вороха, в результате чего значительно снижаются посевные и продовольственные показатели качества зерна. Для устранения этого негативного явления зерновой ворох обрабатывают зернометателями 3М-60, 3М-60А, зернопогрузчиками 3Э-100, ЗПС-100 и другими машинами. Однако они имеют ряд существенных недостатков. Для повышения их эффективности актуален вопрос создания зернометателей, работающих на новых принципах, что является важной научной проблемой, решенной в данной работе.

Свежеубранная зерновая масса называется зерновым ворохом, так как очень разнообразна по своему составу. Эта масса имеет высокую засоренность, влажность, различную микрофлору, физиологически очень активна и её нельзя хранить. Свежеубранное зерно в кратчайшие сроки следует очистить от примесей и высушить до кондиционных параметров [1]. При этом следует учитывать, что послеуборочная обработка зерна является достаточно ресурсоёмким процессом, поэтому правильная его организация обеспечивает сокращение потерь урожая, повышение качества выполнения технологических операций, снижение трудовых, материальных и энергетических затрат. Обработка зерна должна осуществляться на основе комплексной механизации всех согласованных между собой технологических процессов, с применением более совершенных поточных технологий, современных сушильных и зерноочистительных агрегатов, высокой организации труда [2].

Цель работы: создание и внедрение зернометатель-классификатора для предварительной очистки зернопродуктов от мелких, крупных и легких примесей, позволяющая повысить производительность сепараторов и эффективность обработки зерна, вывод на рынок отечественного «Зернометательклассификатор для предварительной очистки зерна». Научная гипотеза. Совмещение предварительной очистки зернового вороха с его подсушкой и охлаждением, позволяющее значительно улучшить качественные показатели зерна путем совершенствования рабочего процесса зернометателей.

Научная новизна работы заключается в комплексном исследовании факторов, влияющих на совершенствование рабочего процесса зернометательных машин, анализе и обобщении теоретических положений и закономерностей, в результате которых:

-разработан «Зернометатель-классификатор ЗКС» нового поколения;

-дано обоснование его конструктивных и кинематических параметров; Новизна предложенных технических разработок подтверждена Инновационными патентами РК на полезную модель [3].

Практическую ценность работы представляют:

-оригинальная структура классификации зернометательных машин.

-обоснованы кинематические и конструктивные параметры зернометателя; 
-предложены новые конструктивные решения при разработке зернометателей;

-разработанные модели без значительных дополнительных затрат и усилий вливаются в существующие технологические линии;

-конструктивные особенности предлагаемых разработок позволяют модернизировать существующие зернометатели;

-дано экономическое обоснование усовершенствованной конструкции зернометателя.

Исследования по послеуборочной обработке зерна ведутся в ИП «Казакбаев С.3.» в течение длительного времени. Учёные научноисследовательской лаборатории (НИЛ) Таразского инновационно-гуманитарного университета (ТИГУ) внесли очень большой вклад по разработке инновационной техники и технологии послеуборочной обработке зерна (ИТТПОЗ), совершенствованию существующих и разработке новых зерноочистительно-сушильных машин, которые внедрены в хозяйствах Жамбылской области.

В настоящее время разработка инновационной техники и технологи по послеуборочной обработке зерна осуществляется индивидуально для каждого хозяйства с учётом вида обработки зерна, природно-климатических и хозяйственных условий, финансовых возможностей. Технологическая процесс ИТТПОЗ может состоять как из отечественных, так и из импортных зерноочистительных машин [4]. Однако, экономически оправданным, что подтверждает практический опыт, является совмещение в одной мобильной машине нерегрузочных операции с технологическими, как очистка зерна от мелких, крупных, металломагнитных, легких примесей и пыли, а также обеззараживание и подсушка зерна.

При разработке ИТТПОЗ и вводе в эксплуатацию хорошо зарекомендовал себя «Зернометатель-классификатор ЗКС», который представляет собой современную машину надёжной и прочной конструкции. «Зернометатель-классификатор ЗКС» предназначен для предварительной, первичной очистки семян зерновых, зернобобовых и масленичных культур и отвечает высоким эксплуатационным требованиям. Он легко поддаётся настройке и может работать с разной производительностью. Например, при обработке пшеницы её паспортная производительность выглядит следующим образом: при предварительной очистке - 60 т/час, при первичной (товарной) очистке - 40 т/час.

Следует учитывать, что фактическая производительность ЗКС отличается от паспортной, зависит от засорённости и влажности зерна. Влияние засорённости и влажности на производительность зерноочистительных машин, входящих в технологическую линию, разное и определяется экспериментальным путём. Так, например, каждый процент увеличения влажности зерна влечёт снижение производительности зерноочистительных машин как при предварительной, так и при первичной очистке в среднем на 5\%, а каждый процент засорённости снижает производительность машин при предварительной очистке на $2 \%$, а при первичной очистке - на 4\%. При этом происходит увеличение затрат на обработку [5].

Очистка зерна от примесей «Зернометательклассификатором ЗКС». Зернометательклассификатор отделяет из поступающей зерновой смеси мелкие, крупные, металломагнитные, легкие примеси и пыли, а также насекомые.

Основные задачи экспериментальных исследований:

Определение оптимальной толщины поступающего слоя зерна и частоты вращения кольцевого ротора, при которых отделяются крупные примеси и создание равномерного распределения расслоенного зерна по площади поперечного сечения пневмосепарирующей камеры.

Определение оптимальной толщины поступающего слоя зерна, которая расслаивается от кольцевого ротора и длины всасывающего жалюзного патрубка, при которых достигается максимальная эффективность очистки зерна от легких примесей.

\section{Опытная установка}

Для оптимизации параметров рабочих органов «ЗКС», технологических режимов процесса очистки зерна от крупных и легких примесей в ПРК и выбор параметров ПРК изготовлена опытная установка, показанная на рис 1.

Опытный «Зернометатель-классификатор ЗКС» работает следующим образом. Продукт, с загрузочного патрубка 1 с электромагнитами 3 , через распределительные клапана 2 поступает на вращающийся кольцевой ротор 4. При этом крупные частицы продукта, оставаясь на поверхности колец 9, через скребок 5 направляются в патрубок 6 для крупных примесей. Застрявшие между кольцами крупные частицы продукта отделяются скребком 5. Для очистки внутренней полости кольцевого ротора и регулирования зазора между кольцами предусмотрены сервисные люки 18 , расположенные в торцевой части корпуса классификатора на уровне кольцевого ротора. Основная часть продукта, перемещаясь вниз, проходит через продольные зазоры, образованные по окружности кольцами 9, 
поступает в пневмосепарирующую камеру 7. При этом общий поток продукта расслаивается и распределяется на небольшие слои, между которыми образуются свободные межзерновые пространства. В вертикальной пневмосепарирующей камере 7 продукт продувается в поперечном направлении воздухом. Воздух при этом входит через регулируемую вертикальную жалюзийную решетку 15, пронизывает свободные межзерновые пространства, выделяет легкие частицы, в том числе пыль, насекомые и направляется через всасывающий пирамидальный патрубок 14 вентилятора в циклон-отделитель, где происходит отделение легких частиц от пылевоздушной смеси. Очищенные частицы продукта с пневмосепарирующей камеры 7 через разгрузочный патрубок 8 поступают на бесконечную ленту триммера.

Пирамидальная форма всасывающих патрубков 14 и регулируемых вертикальных жалюзийных решеток 15 обеспечивает равнораспределенность скорости воздуха внутри пневмосепарирующей камеры 7, что позволит повысить эффективность процесса разделения продукта от легких примесей и пыли. Для визуального наблюдения за процессом сепарации предусмотрены смотровые окна 16, $\begin{array}{lll}\text { расположенные в торцевой части } & \end{array}$ пневмосепарирующей камеры. На Рис.2 изображен общий вид зернометателяклассификатора ЗКС.

Новым в разработке является то, что пневмороторный классификатор установлен на зернометателе между загрузочным транспортером и триммером, что позволит совместить перегрузочные операции с технологическими, как очистка зерна от крупных, металломагнитных, легких примесей и пыли, а также как обеззараживание и предварительная сушка зерна. Кольцевой ротор с установленными внутри него межколечными регуляторами зазора является просеивающим элементом, что способствуют продукту расслаивается на множество слоев и образования свободных межзерновых пространств. При этом вертикально установленные регулируемые воздушный поток жалюзные решетки способствуют целенаправленному и эффективному воздействию воздуха на легкие примеси в вертикальной пневмосепарирующей камере. Вильчатый скребок не только отделяет крупные примеси с поверхности кольцевого ротора, но и предотвращает засорение частиц между кольцами. Для очистки внутренней полости кольцевого ротора и регулирования зазора между кольцами предусмотрены сервисные люки. [6].

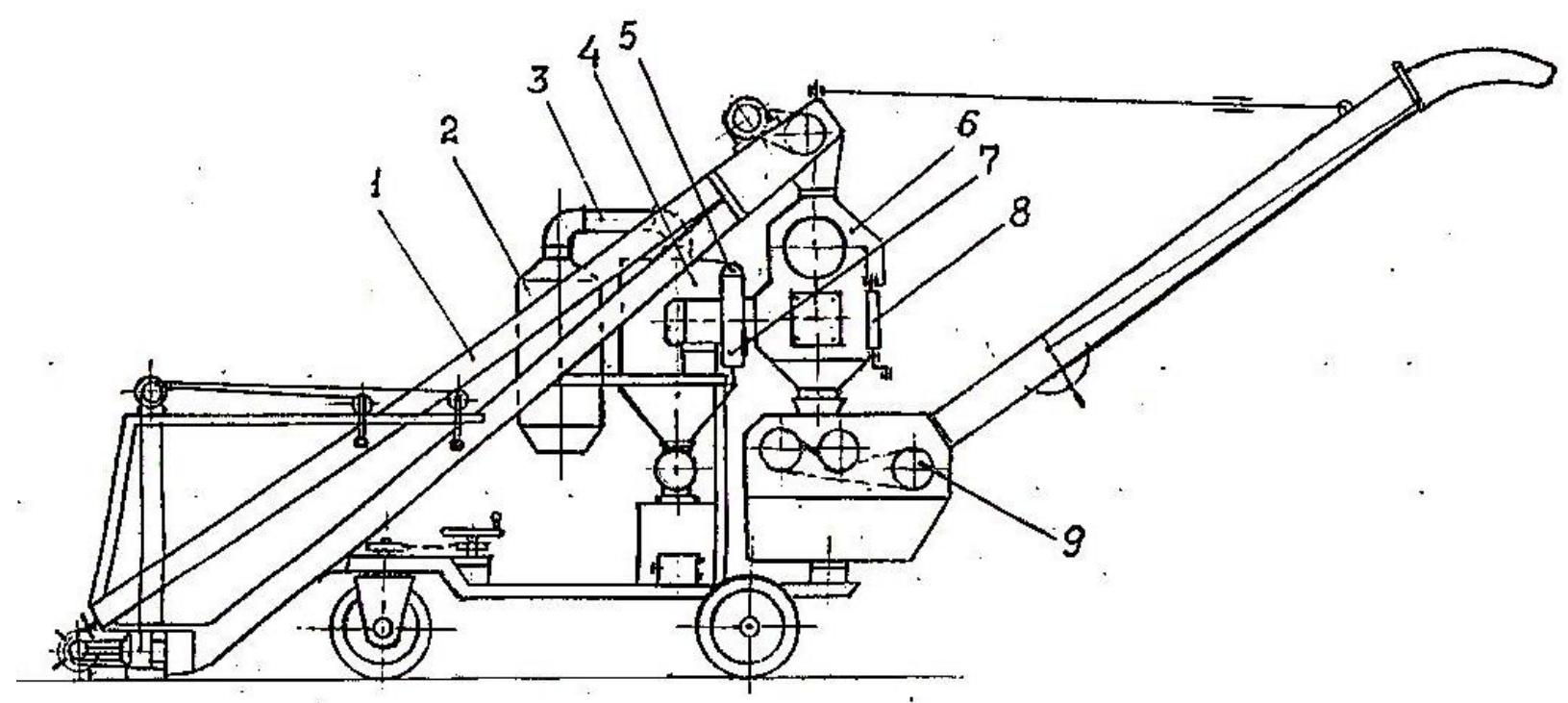

Рисунок 1 - Принципиальная схема зернометателя-классификатора ЗКС. 


\begin{tabular}{|c|c|c|c|c|c|c|}
\hline Impact Factor: & $\begin{array}{l}\text { ISRA (India) } \\
\text { ISI (Dubai, UAE } \\
\text { GIF (Australia) } \\
\text { JIF }\end{array}$ & $\begin{array}{l}=1.344 \\
=0.829 \\
=0.564 \\
=1.500\end{array}$ & $\begin{array}{l}\text { SIS (USA) } \\
\text { PИНЦ (Russia) } \\
\text { ESJI (KZ) } \\
\text { SJIF (Morocco) }\end{array}$ & $\begin{array}{l}=0.912 \\
=0.234 \\
=1.042 \\
=\mathbf{2 . 0 3 1}\end{array}$ & $\begin{array}{l}\text { ICV (Poland) } \\
\text { PIF (India) } \\
\text { IBI (India) }\end{array}$ & $\begin{array}{l}=6.630 \\
=1.940 \\
=4.260\end{array}$ \\
\hline
\end{tabular}

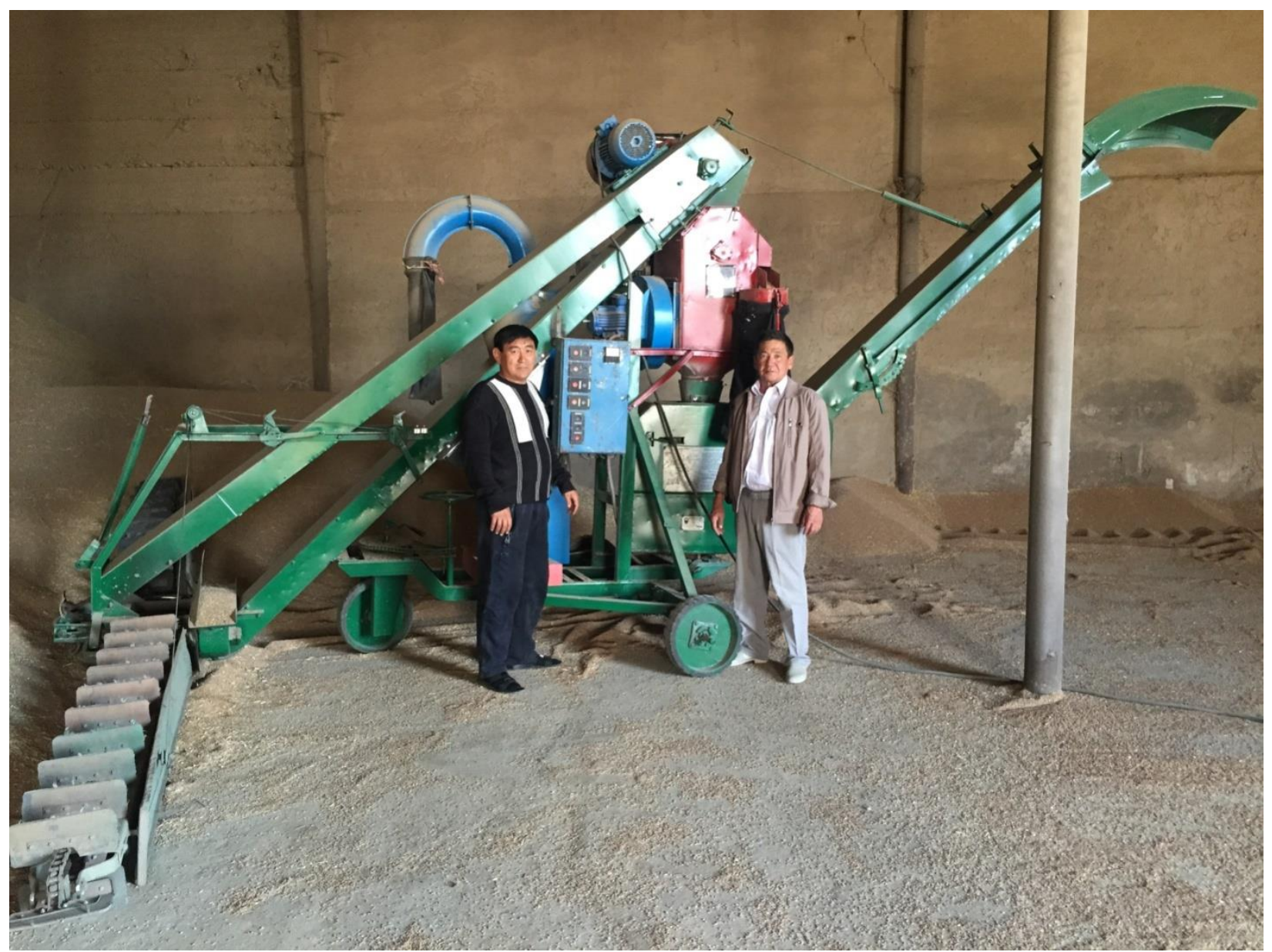

Рисунок 2 - Общий вид зернометателя-классификатора ЗКС.

Пневмороторный классификатор (Рис.3) кроме зернометателя можно установить и в пунктах перегрузки подьемно-транспортных машин: например из бункера на конвейер, с конвейера на ковшовый элеватор и т.д. Это позволит выполнить очистку зерна от крупных, металломагнитных, легких примесей и пыли, а также от вредителей хлебных запасов непосредственно в процессе приемки и транспортирования продукта. При этом сокращаются капитальные и эксплуатационные затраты на приемку и обработку продуктов.

$$
\text { Таким образом, преимуществом }
$$
предлагаемого зернометателя-классификатора ЗКС являются высокие производительность и эффективность очистки зернопродуктов от крупных, металломагнитных, легких примесей и пыли, а также совмещение траноспортных операций с технологическими, такими как обеззараживание зерна, предварительная сушка. Отличительной особенностью ЗК от аналогов является установленная на нем систама, позволяющая управлять с пульта управления.Предлагаемый зернометательклассификатор ЗКС по сравнению с прототипом обеспечивает улучшение условий эксплуатации за счет совершенствования удобства и сервисного обслуживания машины.

Объекты и методы исследований.

Объектами исследований являются зерно пшеницы сорта Алмалы, Стекловидная 24, Богарная 56, ячменя сорта Байшешек урожая 2015 года и аэродинамические примеси применительно к процессу пневмосепарирования и оборудование для осуществления этого процесса.

Опытные исследования выполнены на специально созданных экспериментальных установках и опытных образцах новых пневмороторных классификаторах ПРК, в производственных условиях линии приёма и обработки зерна в крестьянском хозяйстве «Таукебаева С.С.» 


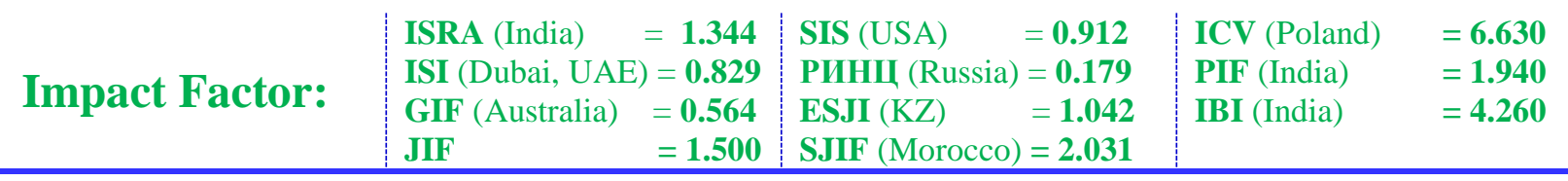

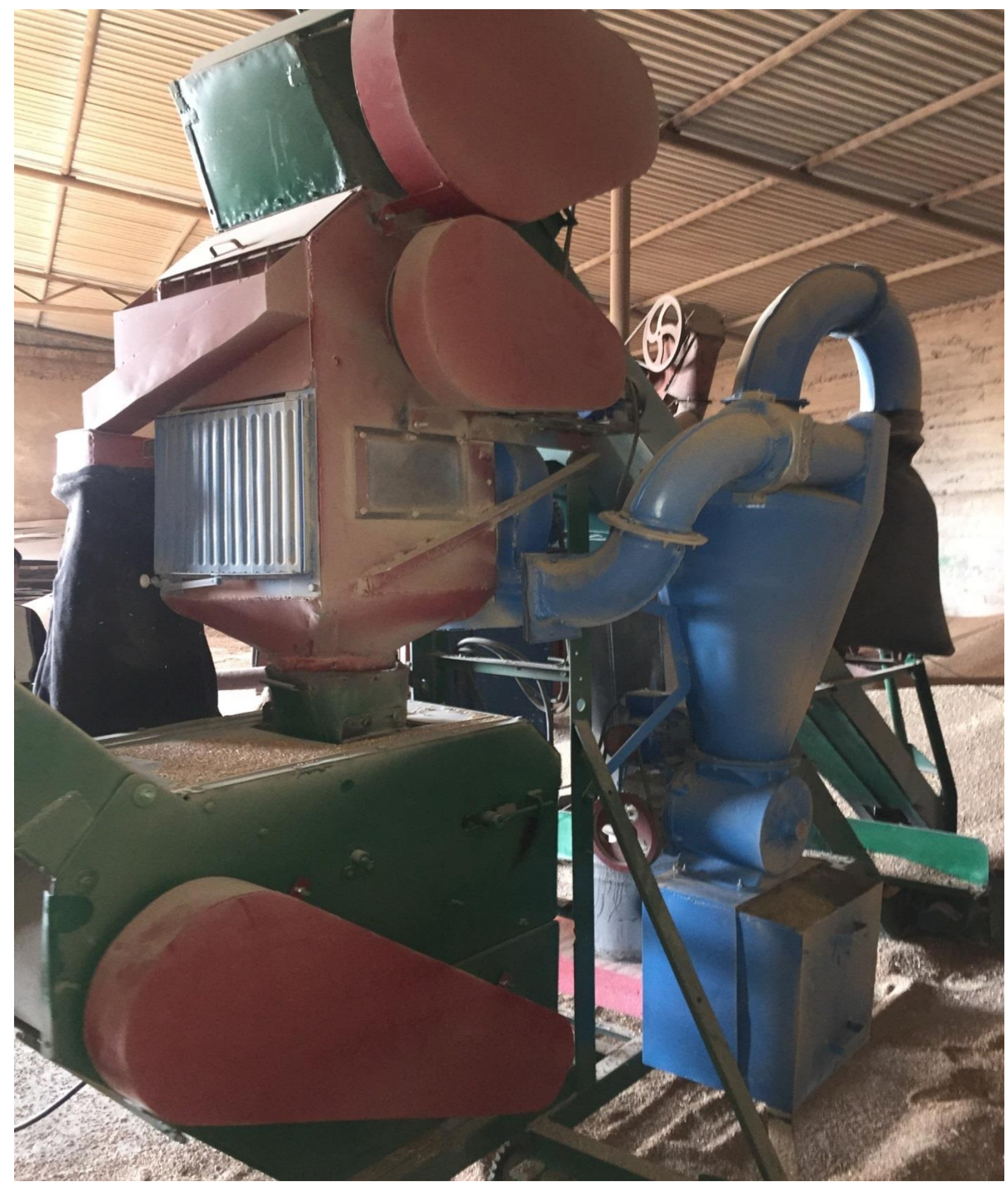

Рисунок 3 - Пневмороторный классификатор.

Обработка результатов экспериментальных исследований выполнена графоаналитиеским методом и на компьютерных программах. В работе использованы методы математической статистики, математического моделирования.

Эксперименты проводились в два этапа.

Первый этап заключался в определении рациональной частоты вращения ротора, количества поперечных пластин внутри колец и вала ротора в зависимости от толщины поступающего слоя зерна, при которых обеспечивается равномерное распределение потока зерна и образуются свободные воздушные прослойки внутри сепарирующей камеры. Как известно, достаточное пространство в зерновом
ISPC Global Science,

Lancaster, USA 
потоке способствует эффективному уносу лёгких примесей воздухом.

В результате первого этапа экспериментальных исследований установлено, что большой коэффициент равномерности распределения зерна $K p=80-92 \%$ достигается при частоте вращения кольцевого ротора $\Pi_{\mathrm{p}},-30$ 40 об/мин и толщины поступающего слоя $\mathrm{h}_{\text {сл }}=$ 30 -50 мм.

Второй этап заключается в установлении рациональной рабочей длины всасывающего жалюзного патрубка в зависимости от толщины поступающего слоя зерна, при котором также будет обеспечиваться максимальная эффективность очистки зерна от лёгких примесей.

На втором этапе эксперимента приняты следующие значения факторов: толщина поступающего слоя зерна $\mathrm{H=30}$ - 70 мм; рабочая длина всасывающего жалюзного патрубка $\mathrm{L}=$ 120 - 400 мм.

В результате экспериментальных данных выявлено, что при расстоянии между кольцами, равном максимальному размеру двух-трёх зерновок (12 мм) и при вращении кольцевого ротора с частотой вращения $n_{p}=25-45$ об/мин крупные примеси, размеры которых превышают зазор между кольцами, полностью отделяются из поступающего зернового слоя, что весьма важно для последующих технологических операций.

Таким образом, преимуществом предлагаемого зернометателя-классификатора
ЗКС являются высокие производительность и эффективность очистки зернопродуктов от крупных, металломагнитных, легких примесей и пыли, а также совмещение траноспортных операций с технологическими, такими как обеззараживание зерна, предварительная сушка. Предлагаемый зернометатель-классификатор ЗК по сравнению с прототипом обеспечивает улучшение условий эксплуатации за счет совершенствования удобства и сервисного обслуживания машины.

Реализация настоящего проекта, позволит заявителю сокращение приведённых затрат в 2,5 раза на 1 тонну обрабатываемого зерна, предотвратить зараженность зерна, произвести предварительную сушку зерна, создать благоприятные условия для сушки и хранения зернопродуктов. Применение предлагаемой техники и технологии позволяют получить экономический эффект 1,5 млн. тенге, при переработке 1000 тонн зерна за счет снижения затрат от совмещения процесса приема и первичной переработки зерна. Использование предлагаемой технологии, помимо очистки от вредных (вегетативных, составных частей ворохи зерна и сорных примесей) примесей, сопровождается продувкой, т.е. предварительной сушкой, что положительно влияет на сохранность и на обеззараживание вредителей хлебных запасов.

\section{References:}

1. (2009) ACE.No 65792 on the invention of PK, from 04.08.2009

2. (2010) ACE.No68849 on the invention of PK, from 01.06.2010

3. (2016) ACE.No 70124 on the invention of PK

4. Demski AB, Vedenev VF (1978) Basic directions pnevmoseparatsionnogo tence of winnowing equipment is accomplished. Moscow: TsNIITEILegpischemash, 1978. -73p.

5. Devidson I, Harrison D (1974) Pseudofluidizing. - M., Chemistry, 1974.-430 p.

6. Zlochevskiy VL (1986) Intensification of the process of aerodynamic separation of granular materials. - Dis. doctor tehn. Sciences. Novosibirsk, 1986 - with 473 p.
7. Zuev FG, Abdeliev DD, Kazahbaev SZ (1990) branch of light impurities from grain products. Journal of agricultural science of Kazakhstan. 1990.-No 6. -pp. 110-115.

8. Karpov BA (1987) Technology postharvest processing grain storage. -Moscow. Agropromizdat, 1987. -288 p.

9. Punkov SP, Iztayev AI (1982) post-harvest processing grains. -Almaty: Kainar, 1982, 167 p.

10. Razvorotnev A (1987) grain dust collection with acceptance. Flour silo industry and mixed feed industry. -1987. - No 4, pp.19-21. 